\title{
The Research on the Affect of Collapse State and Bearing Capacity of the Soil Layer Properties Adjacent to the Bearing Plate of the Multi-Extruded- Expanded-Plates Pile
}

\author{
Yongmei Qian* and Xiaolong Liu
}

Jilin Jianzhu University, Changchun, China

\begin{abstract}
This paper introduces the research status and analysis of the Multi-Extruded-Expanded-Plates pile (the MEEP pile) at home and abroad, by studying when the plate of pile embedded in different characteristics soil layer, the thickness, physical properties of upper and lower soil layer adjacent to the soil with plate cause the influence on soil break and bearing capacity of the soil layer with plate, analyzing the work together with pile and soil in different soil layer, providing a theoretical basis to improve the calculation formula of bearing capacity of single pile, ensuring the rationality and reliability of the design of the MEEP pile.
\end{abstract}

Keywords: The multi-extruded-expanded-plates pile, soil layer thickness, the physical properties of soil, the bearing capacity.

\section{INTRODUCTION}

The research on the MEEP pile [1] under the vertical force already had preliminary results, but the research is mostly carried out in single layer, this paper aiming at this situation, by studying the plate embedded in different characteristics soil layer, the thickness and physical properties of upper and lower soil layer adjacent to the soil with plate cause the influence on soil break and bearing capacity of the soil layer with plate, analyzing the work together with pile and soil in different soil layer, after fully considering the impact, on the basis of existing theory, improving the formula of bearing capacity of single pile.

\section{THE RESEARCH STATUS AND ANALYSIS OF THE DIRECTION}

The MEEP pile is a new pile foundation in recent years, it is in order to fully tap the foundation soil bearing potential of various hard soil layer, set bearing enlarging disk in different parts of the pile body, by increasing the end resistance of disks to improve the compressive or anti pulling force of pile, with high bearing capacity of single pile, material consumption, simple construction and short time limit, etc.

For now, the MEEP pile as bearing pile in engineering has been widely applied and the compression theory is relatively perfect, the MEEP pile under vertical loads of the compression and tension properties and mechanism of failure also detailed research, the effect of piles and soil under the vertical force and damage of soil properties function curve has been derived through experiment and theory, the MEEP pile under the condition of single plate

\footnotetext{
*Address correspondence to this author at the School of Jilin Jianzhu University, Jilin, 130118, P.R. China; Tel: 13504405206;

E-mail: 654675316@qq.com
}

with single plate the vertical uplift and compression bearing capacity formula has been deduced [2].

However, the destruction characteristics of the MEEP pile in different soil layer, study on the reasonable range or design method of qualitative or quantitative determination of related parameters are also relatively few. Previous theory research of the MEEP pile under vertical loads are mostly concentrated in a single layer [3], assuming that pile plate embedded soil layer thickness is infinite, while the actual engineering is many different characters of soil and the thickness of each layer soil are certain limits, when the pile embedded in different characters of soil, the physical properties and thickness of the upper and lower soil whether have impact on the soil layer where the disks in is without research, if it has effect, the vertical force of the existing bearing capacity calculation formula is not accurate enough and its influence factor also needs to be corrected. Therefore, the study of the influence of the bearing disk in different characters of soil needs to urgent research to perfect theoretical foundation.

\section{INFLUENCE OF ADJACENT SOIL PROPERTIES TO THE MEEP PILE}

\section{Effects of Physical Properties and Thickness of Adjacent Soil to Soil Damage}

When the MEEP pile under the vertical force, the soil failure can be divided into the sliding failure and the punching failure [4], the curve of punching failure and sliding failure of single soil layer has been obtained through theoretical analysis and test. But the study is to assume the thickness of soil layer where the disk in is infinite, the development of punching and slippage curve. According to the predetermined trend. When the characters of soil is different, because the different thickness and physical properties of soil adjacent soil where the disk in caused 
different effects on the soil failure punching shear slip curve trend. The different characters of soil contacts surface blanking slip curve turning point or change trend, but the specific development trend remains to be further research and analysis to determine. Now on the different characters of soil, the physical properties and thickness of adjacent soil of disk change the development trend of slip curve prediction as follows:

A of Fig. (1) is the development status of the sliding failure curve of pile compressed, $b$ is the prediction of development status of the slip line when the physical properties of adjacent soil blow the disk changes (The dotted line of layer 3 in the figure is the predicting status of slip lines ), $\mathrm{c}$ is the prediction of development status of the slip line when the thickness of adjacent soil blow the disk changes(The dotted line of layer 3 in the figure is the predicting status of slip lines, the dotted line blow layer 3 is variation of thickness).
A of Fig. (2) is the development status of the punching failure curve of pile pulled out, $b$ is the prediction of development status of the failure curve when the physical properties of adjacent soil above the disk changes (The dotted line of layer 2 in the figure is the predicting status of failure curve), $\mathrm{c}$ is the prediction of development status of the failure curve when the thickness of adjacent soil above the disk changes(The dotted line of layer 2 in the figure is the predicting status of failure curve, the dotted line above layer 2 is variation of thickness).

A of Fig. (3) is the development status of the sliding failure curve of pile pulled out, $b$ is the prediction of development status of the slip line when the physical properties of adjacent soil above the disk changes (The dotted line of layer 2 in the figure is the predicting status of slip lines), c is the prediction of development status of the slip line when the thickness of adjacent soil above the disk changes(The dotted line of layer 2 in the figure is the

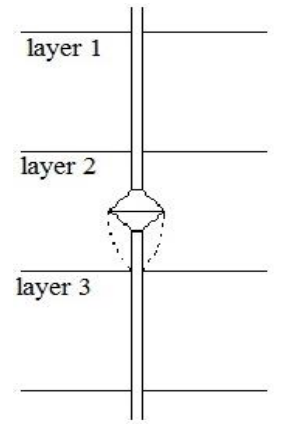

a

Fig. (1). The sliding failure curve of pile compressed.

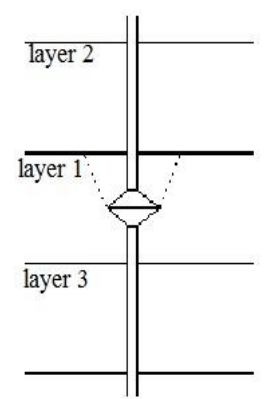

a

Fig. (2). The punching failure curve of pile pulled out.
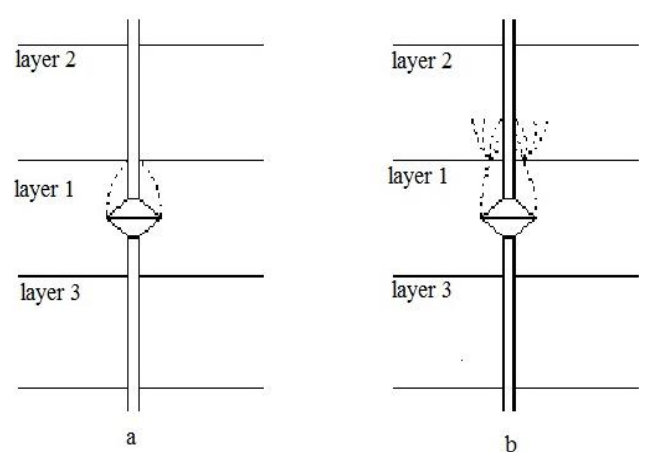

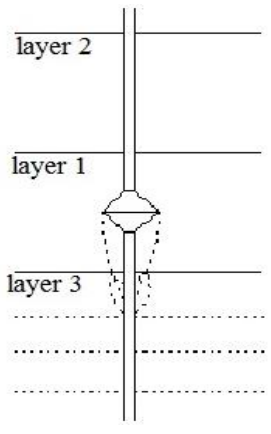

C

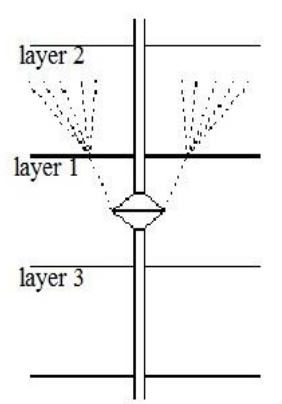

b

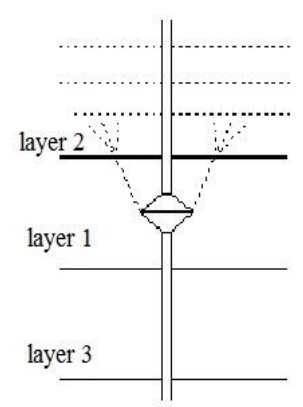

$\mathrm{c}$

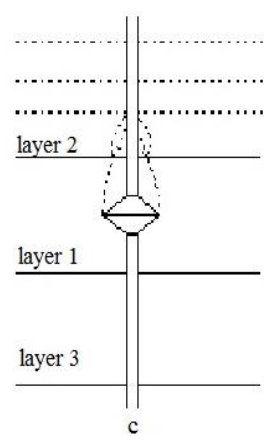

Fig. (3). The sliding failure curve of pile pulled out. 
predicting status of slip lines, the dotted line blow layer 2 is variation of thickness).

A of the Fig. (4) is the development status of the sliding failure curve of pile pulled out, $b$ is the prediction of development status of the slip line when the physical properties of adjacent soil above the disk changes (The dotted line of layer 2 in the figure is the predicting status of slip lines), c is the prediction of development status of the slip line when the thickness of adjacent soil above the disk changes (The dotted line of layer 2 in the figure is the predicting status of slip lines, the dotted line blow layer 2 is variation of thickness).

\section{Effects of Physical Properties and Thickness of Adjacent Soil to Bearing Capacity}

The physical properties and thickness of adjacent soil about the model is assumed as Table $\mathbf{1}$ and Table 2 . The MEEP pile under the vertical force, the bearing capacity calculation model (clay) has also been obtained:

The calculation model of punching failure when it is anti pulling:

$$
\mathrm{P}=\frac{1}{2} c \cos \emptyset p\left(d_{1} l_{1}-d_{2} l_{2}\right)
$$

The calculation model of slip failure when it is anti pulling: $F=\alpha n \pi(D+d) F_{n}+\alpha G+\alpha f l_{0}$

The calculationmodel of slip failure when it is compressive:

$$
\begin{aligned}
& F=\alpha n \pi(D+d) F_{n}+\alpha p_{n} \pi \frac{d^{2}}{4}+\alpha f l_{0} \\
& F_{n}=c \cot \phi\left(e^{2 \theta \tan \phi}-1\right)
\end{aligned}
$$

(In the formula, $c$ and $\phi$ is cohesive force and angle of internal friction of soil)

In the different characteristics of soil, when the MEEP pile under the vertical pull force, the soil above the disk is compressive, in the formula, $c$ and $\phi$ is not only related to this layer of soil, saturation and shear wave velocity of adjacent soil above the plate also has an effect on it, the greater of saturation, the higher of water content and the $c$ in calculation model of value also reduces correspondingly. When the shear wave velocity of soil above the plate is different the $c$ and $\phi$ in the formula will change correspondingly. Therefore the bearing capacity calculation of different characters of soil parameters in the model needs to be corrected, but its concrete correction coefficient needs to determine though the specific analysis research. By the same token, the MEEP pile under vertical pressure calculation model subject to further analysis of the research can be perfect.

When the thickness of adjacent soil layer above the disks is different, the influence on bearing capacity will be different. Its concrete correction coefficient is subject to further research and analysis.

\section{CONCLUSION}

Through the preliminary study shows that, the thickness

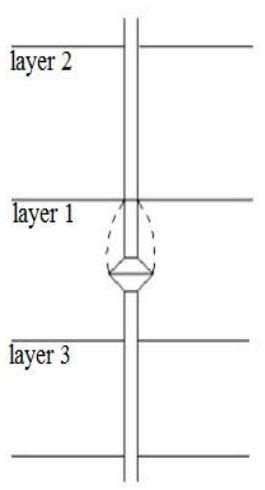

a

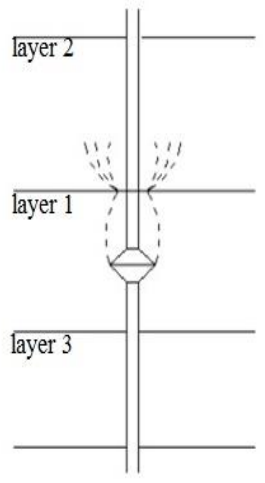

b

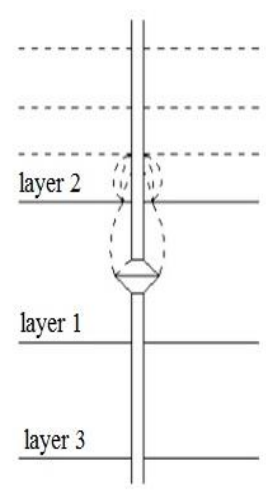

c

\begin{tabular}{|c|c|c|c|}
\hline Saturation $S \mathbf{r}(\%)$ & $S \mathbf{r} \leq \mathbf{5 0}$ & $\mathbf{5 0}<S \mathbf{r} \leq \mathbf{8 0}$ & $S r>80$ \\
\hline Degree of dry and wet & Slightly wet & Very wet & Saturation \\
\hline
\end{tabular}

Fig. (4). The sliding failure curve of pile pulled out.

Table 1. The relationship between degree of saturation and dry and wet.

Table 2. The relationship between shear wave velocity and soil hardness.

\begin{tabular}{|c|c|c|c|c|}
\hline shear wave velocity $V \mathbf{s}$ & $V \mathbf{s}>\mathbf{5 0 0}$ & $\mathbf{5 0 0} \geq V \mathbf{s}>\mathbf{2 5 0}$ & $\mathbf{2 5 0} \geq V \mathbf{s}>\mathbf{1 4 0}$ & $V \mathbf{s} \leq \mathbf{1 4 0}$ \\
\hline \hline Soil soft and hard category & Hard soil or Rock soil & Medium hard soil & Medium soft soil & soft soil \\
\hline
\end{tabular}


and physical properties of upper and lower soil layer adjacent to the soil with plate cause the influence on soil break, which will affect the bearing capacity of pile plate, as for the specific scope of influence and correction coefficient of bearing capacity is still to be further studied. This paper will further improve the compressive and uplift theoretical basis of the MEEP pile under vertical load. Make up the MEEP pile embedded in different characteristics soil layer, the thickness and physical properties of upper and lower soil layer adjacent to the soil with plate cause the influence on soil break and bearing capacity of the soil layer with plate, so as to provide a theoretical basis for the further improvement of the calculation formula of bearing capacity of single pile, to ensure the rationality and reliability of the design of push-extend Multi-under-reamed pile.

\section{CONFLICT OF INTEREST}

The author confirms that this article content has no conflict of interest.

\section{ACKNOWLEDGEMENTS}

This work is financially supported by National Natural Science Foundation of China (51278224).

\section{REFERENCES}

[1] M. S. Wang, D. X. He, and S. T. Tang, "New technology of pile foundation in the 21 st century: DX dig expansion of piles," Engineering Sciences, vol.14, no.1, pp.4-12, 2012.

[2] N. Mu, "Study of the influence about the parameters of the bearing push-extend reamed for the bearing capacity of the tensile resistence of the Push-extend Multi-under-reamed Pile," M.S. Thesis, Jilin Jianzhu University, Chang Chun, China, 2014.

[3] Y. M. Qian, X. S. Yin, R. Z. Wang, and X. X. Yao, "Analysis about the location of the bearing push-extend reamed of pushextend multi-under-reamed pile affecting the bearing capability of the pile," Journal of Jilin Institute of Architecture \& Civil Engineering, vol.27, no.3, pp.1-3, Jun. 2010 .

[4] X. Y. Xie, "Research on Ultimate Bearing Capacity and Relationship between Shear and Displacement of Pile-soil on push-extend Multi-under-reamed Pile," PhD Thesis, Jilin University, Chang Chun, China, 2011. 